\title{
Promise of pharmacogenomics in smoking cessation
}

"...the odds that a smoker who attempts to quit will relapse are still much greater than the chances that he will achieve and maintain abstinence. Improving these odds is a major goal of academic and pharmaceutical therapeutic development programs..."

Individual differences in the ability to quit smoking provide an important model for 'higher order' pharmacogenomics. Twin studies document strong, $c a 0.5$ heritability for success in smoking cessation $[1,2]$. This behavior change yields sizable health benefits. Smoking remains one of the largest preventable causes of death, causing more than 400,000 premature US deaths per year [3]. Many of these deaths could have been deferred by successful midlife cessation, even in individuals who have smoked for years prior to quitting.

While many smokers attempt to quit each year, only approximately one in ten or one in 20 are able to remain abstinent [4]. This rate can be augmented two- or more-fold by treatments that target the brain nicotinic acetylcholine receptors at which nicotine (nicotine replacement or varenicline), or the dopamine transporter (bupropion) exerts its effect. Nevertheless, the odds that a smoker who attempts to quit will relapse are still much greater than the chances that he will achieve and maintain abstinence. Improving these odds is a major goal of academic and pharmaceutical therapeutic development programs, and a promising setting in which thoughtfully applied pharmacogenomics could provide substantial health improvements with favorable cost:benefit ratios.

Pharmacogenomics' promise for smoking cessation includes:

- Enhancing understanding of successful quitting by identifying the genes in which allelic variants contribute to success;

- Providing controls to reduce (genetic) variance and add to power of studies of pharmacological or other environmental influences on quit success;

- Providing controls for stratification (based on quit success alleles) in clinical trials for smoking cessation;

- Allowing clinicians to titrate the magnitude of support for each smoker's quit attempts;
- Personalizing therapies so that the strategy that is most likely to work can be offered to each smoker. Failure to incorporate genetics exerts opportunity costs when an unsuccessful quit attempt follows use of a strategy that does not match the individual smoker or when a trial of a promising smoking cessation medication fails due to unidentified stratification for smoking cessation alleles among trial participants.

\section{"Cost-effectiveness for genotyping in clinical practice will thus improve substantially as genotyping costs drop as our certainty regarding informative markers for smoking cessation success increases..."}

Results from recent genome-wide association studies of smokers who attempt to quit in clinical trial and community settings display gratifyingly high overall degrees of convergence [5-7]. This conclusion springs from the identification of the same chromosomal regions (and genes) by SNPs whose allele frequencies display nominally significant differences between successful versus unsuccessful quitters in each of several independent samples. Genes identified by these clustered, nominally positive results are largely expressed in brain, including many that help to specify neuronal connections and several that are implicated in memory. Elsewhere, we suggest use of primary pharmacogenomics for individual differences in absorption, distribution, metabolism, excretion and toxicology (ADMET), secondary pharmacogenomics for differences in the sites that initially recognize drugs and higher order pharmacogenomics based on individual differences in post-receptor sites that influence drug actions [8]. Most of the genes identified in the smoking cessation work seem likely to alter higher order processes and to influence quitting in individuals treated with nicotine

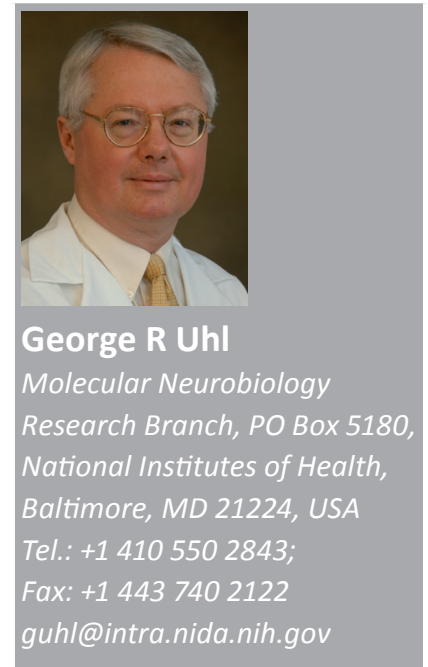

future frotid $_{\text {medicine }}$ fsg 
replacement or bupropion. However, some variants appear to influence responses to only a single treatment type, supporting possible roles for personalized therapeutics as these results are replicated.

These results are also remarkable for what they do not provide. There is no large influence of variants at any particular gene locus. No marker achieves genome-wide significance in any of the four samples for which we have reported genome-wide association data or the three additional samples whose analyses we are completing. The overlap with genome-wide association data from studies of dependence on addictive substances, while significant, is modest. Neither we nor others have identified association between quit success and SNPs in the $\alpha 5$ nicotinic acetylcholine gene cluster that provide the largest current associations with a physiological nicotine-dependence phenotype [9-11]. No current molecular or classical genetic results invalidate additive, polygenic models as first approximations to the genetic architecture of quit success.

\section{"Panels of genotypes that assess higher order pharmacogenomic influences on smoking cessation are increasingly poised to provide significant assistance to smokers who seek to rid themselves of their health-damaging addictions to cigarettes."}

To live up to its promise, the pharmacogenomics of smoking cessation success should address several of the ideas of a recent NHGRI pharmacogenomics white paper: "each individual has a constellation of (risk and protective)...variants...(that should be)...integrate(d) and...use(d)...in the context of (other)...clinical information in ways that (will be) kept costeffective.... and convince (payers to reimburse for them)" [101]. The way forward includes improved documentation of specific sets of smoking cessation success variants and improving understanding of these markers within contexts that include:

\section{Bibliography}

1 Broms U, Silventoinen K, Madden PA, Heath AC, Kaprio J: Genetic architecture of smoking behavior: a study of Finnish adult twins. Twin Res. Hum. Genet. 9(1), 64-72 (2006).

2 Lessov CN, Martin NG, Statham DJ et al.: Defining nicotine dependence for genetic research: evidence from Australian twins. Psychol. Med. 34(5), 865-879 (2004).

3 Adhikari B, Kahende J, Malarcher A, Pechacek T, Tong V: Smoking-attributable mortality, years of potential life lost, and productivity losses - United States, 2000-2004. Morb. Mortal. Wkly Rep. 57(45), 1226-1228 (2008).
- Racial/ethnic background of the individual involved [12];

- Important clinical variables that include degree of dependence (e.g., scores on specific items on the Fagerström physiological dependence test), gender, age and comorbidities;

- Features of the initial smoking cessation effort, such as smoking rates during a prequit period when nicotine replacement may have been initiated.

We have recently documented the striking cost-effectiveness of genotyping participants in medium-sized smoking cessation clinical trials [13]. In this setting both costs per subject and the opportunity cost of false-negative results due to stochastic stratification for quit success alleles are both quite high. It is reasonable to add the costs of a failed quit success attempt to the lost opportunity costs for smokers who become discouraged. Cost-effectiveness for genotyping in clinical practice will thus improve substantially as genotyping costs drop as our certainty regarding informative markers for smoking cessation success increases and as some markers predict better responses to some pharmacotherapies than others.

Panels of genotypes that assess higher order pharmacogenomic influences on smoking cessation are increasingly poised to provide significant assistance to smokers who seek to rid themselves of their health-damaging addictions to cigarettes.

Financial \& competing interests disclosure

This study was supported by the National Institutes of Health - Intramural Research Program, National Institute on Drug Abuse, Department of Health and Social Services. Dr Uhl is listed as an inventor for a patent application filed by Duke University based on genomic markers that distinguish successful quitters from unsuccessful quitters in data from clinical trials. The author has no other relevant affiliations or financial involvement with any organization or entity with a financial interest in or financial conflict with the subject matter or materials discussed in the manuscript apart from the disclosed.

No writing assistance was utilized in the production of this manuscript.

4 Zhu S, Melcer T, Sun J, Rosbrook B, Pierce JP: Smoking cessation with and without assistance: a population-based analysis. Am. J. Prev. Med. 18(4), 305-311 (2000).

5 Drgon T, Montoya I, Johnson C et al.: Genome-wide association for nicotine dependence and smoking cessation success in NIH research volunteers. Mol. Med. 15(1-2), 21-27 (2009). 
6 Uhl GR, Liu QR, Drgon T, Johnson C, Walther D, Rose JE: Molecular genetics of nicotine dependence and abstinence: whole genome association using 520,000 SNPs. BMC Genet. 8, 10 (2007).

7 Uhl GR, Liu QR, Drgon T et al.: Molecular genetics of successful smoking cessation: convergent genome-wide association study results. Arch. Gen. Psychiatry 65(6), 683-693 (2008).

8 Uhl GR, Drgon T, Johnson C et al.: 'Higher order' addiction molecular genetics: convergent data from genome-wide association in humans and mice. Biochem. Pharmacol. 75(1), 98-111 (2008).

9 Saccone NL, Saccone SF, Hinrichs AL et al.: Multiple distinct risk loci for nicotine dependence identified by dense coverage of the complete family of nicotinic receptor subunit (CHRN) genes. Am. J. Med. Genet. B Neuropsychiatr. Genet. 150B(4), 453-466 (2009).

10 Bierut LJ, Madden PA, Breslau N et al.: Novel genes identified in a high-density genome wide association study for nicotine dependence. Hum. Mol. Genet. 16(1), 24-35 (2007).

11 Saccone SF, Hinrichs AL, Saccone NL et al:: Cholinergic nicotinic receptor genes implicated in a nicotine dependence association study targeting 348 candidate genes with 3713 SNPs. Hum. Mol. Genet. 16(1), 36-49 (2007).

12 Rose JE, Herskovic JE, Behm FM, Westman EC: Pre-cessation treatment with nicotine patch significantly increases abstinence rates relative to conventional treatment. Nicotine Tob. Res. (2009) (In press).

13 Uhl GR, Drgon T, Johnson C, Rose JE: Nicotine abstinence genotyping: assessing the impact on smoking cessation clinical trials. Pharmacogenomics J. 9(2), 111-115 (2008).

\section{Website}

101 Applying Genomics to Clinical Problems - Diagnostics, Preventive Medicine, Pharmacogenomics www.genome.gov/27529204 